\title{
Piloting a Smart Rollator: User experiences with technology-related motivation and physical activity
}

\author{
Tage Orenius $\mathrm{PsL}^{\mathrm{a}, *}$, Susanna Paloniemi $\mathrm{PhD}^{\mathrm{b}}$, Heikki Hurri MD PhD ${ }^{\mathrm{c}}$, Olli Kuusisto \\ $\mathrm{MSC}^{\mathrm{d}}$, Leena Ristolainen PT DSc ${ }^{\mathrm{a}}$, Antti Tolonen $\mathrm{MSc}^{\mathrm{e}}$, Anu Seisto PhD ${ }^{\mathrm{d}}$, Heba \\ Sourkatti MSc ${ }^{f}$, Anna Sachinopoulou MSc ${ }^{g}$, Tapio Leppänen SRT ${ }^{d}$, Luc Cluitmans \\ $\mathrm{PhD}^{\mathrm{e}}$, Timo Urhemaa MSc ${ }^{\mathrm{e}}$, Anne Railo-Granfelt PT', Angelos Balatsas-Lekkas \\ $\mathrm{PhD}^{\mathrm{d}}$, Mark van Gils $\mathrm{PhD}^{\mathrm{e}}$
}

\begin{abstract}
${ }^{a}$ Research Institute Orton, Helsinki, Finland; ${ }^{b}$ Department of Education, University of Jyväskylä, Jyväskylä, Finland; ' Orton Orthopaedic Hospital, Helsinki, Finland; ${ }^{\text {VTT Techni- }}$ cal Research Centre of Finland, Espoo, Finland; 'VTT Technical Research Centre of Finland, Tampere, Finland; ${ }^{f}$ VTT Technical Research Centre of Finland, Kuopio, Finland; ${ }^{g}$ VTT Technical Research Centre of Finland, Oulu, Finland; Corresponding author: tage.orenius@orton.fi
\end{abstract}

\begin{abstract}
Background: Improved life expectancy combined with suboptimal physical activity (PA) represents an increasingly salient public health challenge among the elderly. PA in late life is associated with fewer health problems in old age. Assistive information and communication technology might improve PA and alleviate health problems among the elderly.

Objective: This pilot study aimed to quantitatively measure the motivational aspects related to rollator use and, by using qualitative interviews, outline how a Smart Rollator solution would motivate older adults to increase their PA in their everyday lives.

Method: A total of 19 subjects between the ages of 63 and 91 years participated in the study. Half of the participants started in a setting in which the application did not provide feedback to the user, and the other half received feedback. A transition occurred (ordinary rollator to Smart Rollator and vice versa) after two months of usage. Motivational aspects were measured before the use of the rollator and after four months. Semi-structured qualitative interviews were conducted with 10 participants to acquire information about their experiences. Results: On the motivation questionnaire, self-perceived mental vitality showed a significant decrease at follow-up, but the total score did not change. Three different types of Smart Rollator users were identified based on the interview data: enthusiastic, practical, and disappointed users. The user types differed from each other, especially regarding user experiences concerning the smart features and intelligent features of the rollator.

Conclusion: We conclude that the individual variations in terms of benefiting from the use of the Smart Rollator were large and that some users reported clear advantages using the Smart Rollator. The Smart Rollator elicited emotional reactions and affection, as well as frustration if the user was not able to benefit from the Smart Rollator as expected. Larger sample size is warranted to thoroughly specify the relations between the use of a Smart Rollator, user experiences, and PA.
\end{abstract}

Keywords: Rehabilitation, older adults, motivation, Smart Rollator, physical activity

\section{INTRODUCTION}

The increase in life expectancy has posed challenges regarding the health care system's ability to provide necessary health services to older adults while managing the associated expenses (Parker \& Thorslund, 2007; Majumder et al., 2017). According to reports from the World Health Organization (2015), about $60 \%$ of people's quality of life and health depends on their lifestyle and personal behavior, and $53 \%$ of fatalities are associated with lifestyle and health behaviors (Briggs et al., 2016). Physical activity (PA) positively relates to improved functional benefits and positive health outcomes for older adults (Bouaziz et al., 2016) and is strongly associated with all dimensions of health-related qual- ity of life (HRQoL) among community-dwelling people above 60 years old (Halaweh et al., 2015). Everyday PA also has beneficial effects on mortality, even when environmental health factors are considered (Andersen et al., 2015). A dose of moderate to vigorous-intensity PA below current recommendations (i.e., a minimum of 150 min of moderate-intensity or 75 min of vigorousintensity PA per week or an equivalent combination of moderate and vigorous PA) according to The Physical Activity Guidelines Advisory Committee Report (2008) reduced mortality by $22 \%$ among older adults, with a further increase in PA linearly improving these benefits (Hupin et al., 2015). Therefore, older adults who adopt healthimproving behaviors can be healthier in their old 
age (Changizi \& Kaveh, 2017).

Research shows that among older adults, PA, and fear of falling are related to each other (Sawa et al., 2020). In a study by Cruz et al. (2017), the prevalence of fear of falling among older adults was as high as $95.2 \%$ and showed associations with age, self-perceived health, difficulty walking, use of an assistive device for walking, history of falls, and functional capacity. Higher PA levels seemed to be protective regarding fear of falling among both genders (Chang \& Do, 2015). A systematic review (Denkinger et al., 2015) revealed that the parameters robustly associated with older adults' fear of falling were, among others, the use of a walking aid, a history of falls, and poor self-rated health. The relation between the use of walking aids and actual falls can be complex and might even be mediated by various factors. A substantial proportion of the relationship between walking aids and future falls could be explained by an altered spatiotemporal gait pattern, increased age, and psychotropic drug intake (Roman de Mettelinge \& Cambier, 2015).

Assistive technology (AT) could play an important role for older adults in terms of remaining active, but their experiences with electronic technologies are influenced by multiple factors. There is evidence that information and communications technology (ICT) applications can improve lifestyle and health-related behaviors among older adults (Mostaghel, 2016). An understanding of the personal and socio-technical factors of user engagement can help enable the development of technologies that promote both PA and social interaction for older adults (De Angeli et al., 2016). The adoption of technology is a complex issue affected by multiple factors; the use of mobile applications is driven by nonfunctional motives (i.e., social and experiential) and functional motives relating more directly to the use of technology (Reid et al., 2017). The facilitating factors relating to older adults' adoption of technology are value for money, usability, affordability, accessibility, technical support, social support, emotion, independence, experience, and confidence (Lee \& Coughlin, 2015). Mobility devices are used to enable mobility and retain independence in everyday life and for participation in social activities. The use of assistive walking devices facilitates movement and diminishes the risk of falling; when incorporated into daily life, walking aids have been found to enable several domains of activity and participation (Mansouri \& Goher, 2016). Here, an effective rollator design and a more accessible outdoor environment are considered important for optimal use (Brandt et al., 2003).
Rollators equipped with intelligent features can be important devices that aid in the rehabilitation process because they provide useful information for those in charge of rehabilitation (Postolache et al., 2015). Studies show the efficacy of walking aids for older adults with gait changes and imbalance that have developed because of various factors, such as surgery of the lower limbs or neurodegenerative changes, especially in the early recovery period (Postolache et al., 2015). Rollators with intelligent features can make important contributions to improving the quality of life of users by monitoring unbalanced and unstable conditions that can result in falls and injuries (Postolache et al., 2015). The care setting around rollators with intelligent features for older adults must consider the special requirements of the users (Einbinder \& Horrom, 2010), in addition to the viewpoints of their important relations and care personnel. Mere technological solutions are insufficient without understanding the needs of older users and possible use purposes (Martins et al., 2015).

However, older adults might have negative attitudes toward technology. The negative attitudes that are most frequently associated with technologies involve a stigmatizing symbolism that might prevent individuals from adopting the technology (Yusif et al., 2016). Whether walking aids can facilitate activity and participation may depend on the user's ability to overcome obstacles and integrate the aids into their daily lives (Bertrand et al., 2017). Even though older adults represent a growing group of technology users (Vroman, Arthanat, \& Lysack, 2015), not all older adults face rapid changes in intelligent technology in a similar way. Hill, Betts, and Gardner (2015) have reported that the variation in technological skills, attitudes, and experience is wide among the older generations. According to a recent systematic review, there is limited research on rollator-supported gait in older adults (Mundt et al., 2019). Therefore, we need a more comprehensive understanding of the experiences of older adults' intelligent technology use.

\section{Aims AND RESEARCH QUESTIONS}

The current research project aimed to develop a service solution (Smart Rollator and services) that would enable seniors with reduced mobility to move more than part of their everyday lives. A regular rollator was retrofitted with sensors, and feedback information about the amount of movement was given via a smartphone application. The Smart Rollator was evaluated based on comparisons (use of the Smart Rollator with feedback app vs. use without any feedback) and ordinary care situations. 


\section{Pilloting a Smart Rollator}
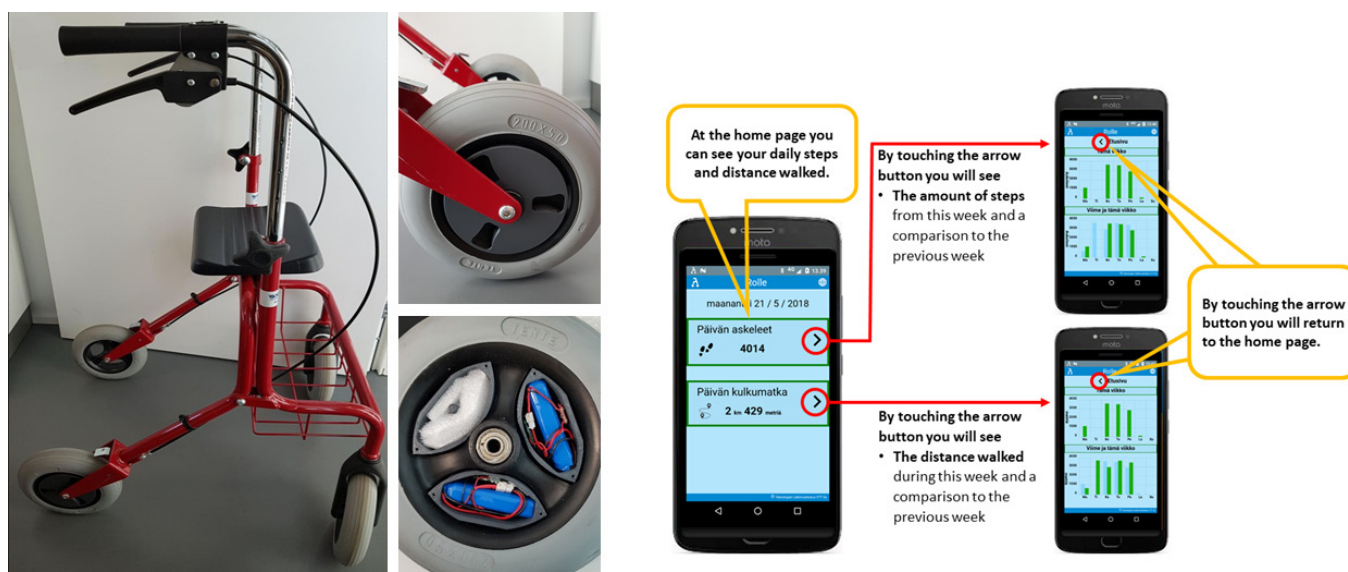

to the home page.

Figure 1. The Smart Rollator, the sensor, and the application.

To date, there has been no study of different user types in older adults using rollators equipped with intelligent features. Therefore, we conducted a pilot study in which we quantitatively measured the motivational aspects regarding rollator use, and by using qualitative interviews, we more outlined how a Smart Rollator solution would motivate older adults to increase their PA in their everyday lives. The research questions addressed in the present study were as follows:

1. To what degree were older adults motivated to use rollators, and what are their experiences using a Smart Rollator?

2. What kinds of Smart Rollator user types can be identified based on older adults' experiences?

\section{Methods AND MATERIALS \\ Participants}

For the entire research project, we recruited 19 participants at or above the age of 60 . The firsttime rollator users ( $n=9 ; 8$ females) had a mean age of 80 (standard deviation [SD] 8.9) years (63-91), and those with earlier experience using a rollator ( $n=10 ; 9$ females) had a mean age of 81 (SD 7.1) years (66-88). There was one exitus (female) during the follow-up in the group of first-time rollator users. The participants were recruited through the medical aid services centers of Helsinki's city hospitals in Finland.

The inclusion criteria consisted of a long-term need for a rollator (i.e., those participants who had been recommended to get one by medical aid services but were still on the waiting list), an ability to engage in independent movement, and sufficient cognitive capacity to use a smartphone as demonstrated by the previous use of a smartphone or the occupational therapist's observation of the ability to use a smartphone. The need for a rollator was evaluated by an occupational therapist at the communal aid care service. If the client needed a rollator to enable movement at home and outside, the client had the right to get a rollator for as long as he or she needed.

The exclusion criteria included life expectancy of fewer than 12 months, reported alcohol misuse, and excessive psychological distress (i.e., expressed fear of moving outside their home, anxiety, feelings of insecurity, etc.) during the meeting with the physiotherapist.

During the recruiting process, we found that the group of participants was heterogeneous in terms of their experience of using a rollator. We were not fully able to recruit first-time rollator users as originally intended, so we also had to include volunteers who had previous experience with a rollator (i.e., participants who had been using one based on recommendations by medical aid services). An interview observation related to this was that as a first rollator, a Smart Rollator might be too demanding: one must become familiar with using a rollator and concurrently be able to apply the additional intelligent features of the rollator. Because of this, the recruitment of participants was extended to include earlier rollator experience. Therefore, because of the heterogeneous group of participants, we measured the motivation of the whole group of participants by using a motivation questionnaire (MQ).

Ten of the participants voluntarily participated in a qualitative follow-up interview study. Of the participants interviewed, six were first-time rollator users, and four had previous experience of using the rollator. Nine participants were women, and the age range was 72 to 91 years of age (mean age 83 years). The participants were interviewed three times (a total of 30 interviews) during the first week of using the rollator and after two and four months of use.

\section{The Smart Rollator}

The VTT Technical Research Centre of Finland developed the prototype of a Smart Rollator, which 


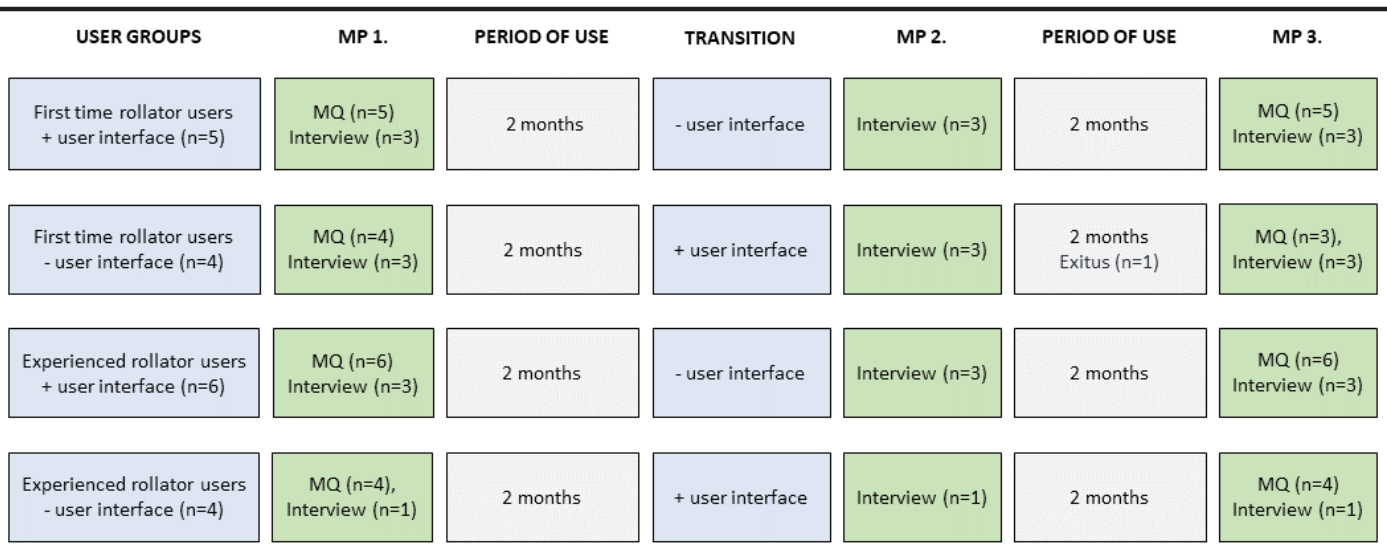

\section{TIMELINE}

Figure 2. Study timeline.

enabled the monitoring of the length of the distance walked and the number of steps on a daily or weekly basis. The intelligent features of the Smart Rollator prototype were applied by using a sensor installed in the rear wheel of traditional rollators. The sensor detected the movement of the wheel and communicated wirelessly with a smartphone that sent motion information to the cloud. The data analysis and visual feedback were provided to the user via the smartphone. The intelligent features were installed using two types of commonly used factory-made rollators: a more robust version called Mauno ${ }^{\circledR}$ and a lighter version called Melody ${ }^{\circledR}$. The Smart Rollator, the sensor, and the application are presented in Figure 1.

\section{Study design}

In this pilot study, a longitudinal crossover study design was utilized. The crossover method enabled a balance between user groups, whereby half of the participants started in a setting in which the application did not provide feedback to the user, and the other half received feedback. A switchover occurred (ordinary rollator --> Smart Rollator, and vice versa) after two months of usage. In the present study, each participant was exposed to this protocol in random order. The total duration of this pilot study was four months. The study timeline is presented in Figure 2.

Abbreviations: $M P$, measuring point; $M Q$, Motivation Questionnaire; User group, four different user groups in the study; Period of use, time (months) of using the Smart Rollator with or without the user interface; Transition, two months from the beginning of the study, a switchover occurred from an ordinary rollator to Smart Rollator and vice versa; MP1, measuring point at the beginning of the study; MP2, measuring point two months from the beginning of the study; MP3, measuring point four months from the beginning of the study, at the end of the study.

\section{Data collection}

The MQ was a custom-made motivation questionnaire assessing the motivational factors related to the use of rollators. It consisted of a total of nine motivation-associated statements, reflecting the attitudes and experiences related to expectations regarding rollator usage in general (items 1-5), as well as statements reflecting internal reward (i.e., intrinsic motivation) factors (items 6 and 7) and external reward (i.e., extrinsic motivation) factors (items 8 and 9).

The MQ was constructed based on current theoretical approaches to human motivation (O'Neil \& Drillings, 2012; Ryan, 2012), with the items tailored to fit the first study question of the pilot study. The items assessing motivation in general and benefit-related factors reflected practical behavioral aspects regarding the use of a rollator. The items were loosely modeled from questionnaires used in earlier studies on motivation (e.g., Glynn et al., 2009; Cocosila et al., 2009). The selection of the items addressing internal and external reward factors was based on background theories about intrinsic and extrinsic motivation (Guay et al., 2000; Reiss, 2004).

Answers were marked on a standard five-point Likert scale ranging from "I completely disagree" to "I completely agree" (1-5). The total scores ranged from 9 to 45, with a higher score indicating a higher level of motivation. No standardization procedure or measurement of validity and reliability was performed. The MQ construct and the results are presented in Table 1 in the "Results" section. The participants completed the $\mathrm{MQ}$ at the beginning of the study and after four months $(2+2)$ of rollator use (Figure 2). 
Table 1. The items of the MQ used in the study, including the results.

\begin{tabular}{lccc}
\hline Items & Baseline & At $\mathbf{4}$ months & Change \\
\hline 1) I'm motivated to use the rollator & $4.5 \pm 0.5$ & $4.3 \pm 0.7$ & $-0.2 \pm 0.9$ \\
2) With the rollator, I will move more outdoors & $4.4 \pm 0.8$ & $4.1 \pm 0.9$ & $-0.3 \pm 1.2$ \\
3) With the rollator, I will do longer walks than without the rollator & $4.2 \pm 0.9$ & $4.1 \pm 1.1$ & $-0.1 \pm 1.5$ \\
4) With the rollator, my fear of falling will decrease & $4.6 \pm 0.6$ & $4.4 \pm 0.8$ & $-0.1 \pm 1.0$ \\
5) With the help of the rollator, my physical fitness will improve & $4.4 \pm 0.8$ & $3.9 \pm 0.8$ & $-0.4 \pm 1.2$ \\
6) With the help of the rollator, my mental vitality will improve & $4.3 \pm 0.5$ & $3.6 \pm 0.8$ & $-0.7 \pm 0.9 * *$ \\
7) My use of the rollator will give me pleasure & $4.2 \pm 0.7$ & $4.1 \pm 0.7$ & $-0.1 \pm 1.1$ \\
8) People (spouse, acquaintances) support my use of the rollator & $3.9 \pm 0.8$ & $4.4 \pm 0.7$ & $0.4 \pm 1.0$ \\
9) People will appreciate my use of the rollator & $3.9 \pm 0.8$ & $3.9 \pm 1.0$ & $0.1 \pm 0.8$ \\
Total scores & $38.2 \pm 4.4$ & $36.9 \pm 4.1$ & $-1.3 \pm 5.5$ \\
\hline
\end{tabular}

** $\mathrm{p}<0.01$

Abbreviation: $\mathrm{MQ}$, motivation questionnaire

The qualitative interviews aimed to address the participants' experiences of using the Smart Rollator in their daily lives, their perceptions of the intelligent features of the rollator, and their self-reports concerning the role of the Smart Rollator in motivating them to move. Furthermore, the possible changes throughout the pilot study were examined.

The semi-structured interview guide, which was developed by two rehabilitation psychologists, included the following themes: (i) quality of life (e.g., ability to function, self-perception of mental vitality, independence in daily life, life satisfaction), (ii) experiences using a rollator (e.g., implementation of the rollator, possible changes in experiences with rollator use, challenges experienced, estimation regarding using the rollator in the future), (iii) experiences with the intelligent features of the Smart Rollator (e.g., previous experiences using the intelligent features, possible challenges faced, the meaning of intelligent features in terms of motivation to move, etc.), and (iv) suggestions concerning the development of the Smart Rollator. The semistructured interview guide frame was developed in line with the research questions by focusing on the individual descriptions of daily life and experiences related to the use of the rollator. The questions reflected practical and clinical experience as well, with the background framework borrowing from different research fields, such as school motivation (Henry \& Thorsen, 2018), health behavior change (Morton et al., 2015), and PA (Farholm \& Sørensen, 2016).

The interviews were conducted by a physiotherapist at the participants' homes. The total duration of the interviews was approximately 30 minutes per participant (range 15-50 min). The recorded interview data were transcribed verbatim.

\section{Questionnaire data analysis}

Changes in MQ scores between different measurement points were tested using the Wilcoxon labeled position number test. Changes were tested in terms of the differences in the nine motivation-related items and MQ total score between baseline and at the four-month follow-up.

\section{Interview data analysis}

A qualitative content analysis (Braun \& Clarke, 2006) and narrative analysis (Riessman, 2008) were applied to the interview data. The data analysis was carried out using the MS Word "find" features (e.g., organizing the data according to the content) combined with manual paper and pencil techniques (e.g., different colors used for different codes). The analysis was conducted in line with the second research question of the study and consisted of three interrelated phases. First, in the data reduction phase, the qualitative codes and data themes were identified. The identified themes included the implementation of the rollator, means of using the rollator, pros and cons of using the Smart Rollator in daily life, attitudes toward and interest in the intelligent features of the Smart Rollator, experiences and feelings regarding the intelligent features of the rollator, the role of the Smart Rollator in motivating physical movement, and prospects concerning the Smart Rollator.

Second, narrative stories regarding each participant were constructed according to the themes identified in the first phases. In this process, the follow-up timeline was utilized. Thus, the sto- 
ries were organized according to the three-point timeline of the study design, that is, in the implementation of the traditional or Smart Rollator after two months in the transition phase (from traditional to Smart Rollator or vice versa) and after four months at the end of the pilot. The focus of the follow-up perspective was to address the possible change in the participants' experiences, motivation, and feelings of using the Smart Rollator.

In the third phase of the analysis, Smart Rollator user types were identified and described based on the narrative stories constructed. Here, the process of constant comparison of similarities and differences was applied in identifying the user type patterns across the interviews (Braun \& Clarke, 2006).

\section{Results \\ Motivation}

The total motivation score of the MQ did not change between baseline and four-month followup. However, a significant $(p<0.01)$ decrease was found in self-perception of mental vitality at the four-month follow-up. The MQ scores are presented in Table 1.

\section{Smart Rollator user types}

The analysis of the interview data resulted in three different user types, each of which was associated with a collection of main features and possible changes in experiences of using the Smart Rollator. The three different user types were enthusiastic, practical, and disappointed users.

Right from the beginning, enthusiastic users were interested in participation and the intelligent features of the Smart Rollator. They were eager to learn new things and to do their part in the research. Despite the challenges faced during application use, they were excited to follow the mobility distance feedback that triggered their activity. One of the participants said, "The feedback really motivates me to move more. It is exciting to see how far I have gone". Overall, enthusiastic users developed a positive and enthusiastic relationship with the Smart Rollator that was sustained for the entire time.

Practical users described their experiences in terms of practical matters. Because their life situation had changed, they were now in need of a rollator, especially to complete daily tasks and ensure safety in independent outdoor mobility. The intelligent features were not meaningful or interesting for them although they were pleased to participate in the pilot study. Thus, for some practical users, participation in the project motivated them the most. Practical users were in charge of using the rollator and exhibited a great deal of agency in deciding when and where they used the rollator. One of the practical users described this as follows: "I go with the rollator only to those places that I know is possible".

Disappointed users were interested in the project and excited about the intelligent features of the rollator at the beginning of the project. However, they faced technical challenges and problems when using a smartphone and the application. For example, they were not able to find the feedback information from the application, or the application did not work properly because the users forgot to carry the smartphone with them. They experienced disappointment and frustration, thus feeling that they were not sufficiently competent to use the Smart Rollator. These users were disappointed because their expectations of the Smart Rollator were not met. In the interviews, these users described their relationship with the Smart Rollator with strong negative emotions, as is apparent in the following extract: "I got angry with it, because it didn't show me information it should have shown". In one case, the participant stopped using the Smart Rollator because of the problems faced.

Overall, Smart Rollator had several purposes and functions in the everyday lives of the participants. For those participants who had recently faced a change in their life situation and who were first-time rollator users, the Smart Rollator was a welcomed aid for their autonomous outdoor mobility. Further, the benefits of PA and motivation to move more were related to several aspects of the research project. First, the feedback from the application was meaningful, especially for enthusiastic users, and this feedback increased their motivation to move more. Here, the positive experiences of using the technology resulted in emotions of joy and excitement. Second, being part of the pilot study was a meaningful experience. In this case, the participants wanted to do their best, and thus, they reported increased activity themselves. This was especially the case with practical users. Third, if the user was not able to benefit from the Smart Rollator as expected, frustration, irritation, and disappointment were reported. These emotions were most often experienced and reported by the disappointed users.

\section{Discussion}

In the current pilot study, we evaluated older adults' use of the Smart Rollator compared with an ordinary rollator. We quantitatively analyzed the motivation regarding the use of rollators. In addition, we addressed participants' experiences using a qualitative interview to explore Smart Rollator user types regarding the effects of the Smart Rollator solution on their motivation to increase PA in their daily lives. 
The results indicated no significant change in the MQ total score at the follow-up after four months (MP3). Only the statement regarding selfperception of mental vitality was associated with a decrease at follow-up; this surprising finding calls for interpretations. Participation in the study may have altered the behavior of the participants because of an initial activation-related increase in the participants' self-perception of mental vitality, manifesting itself in the scores of the first measuring of the MQ. This behavior change has also been described as the Hawthorne effect (McCambridge et al., 2014), according to which knowledge of participating in an experiment modifies the behavior of the participant from what it would have been without this knowledge. However, it remains unclear whether this effect would have affected the self-perceived mental vitality only without influencing the other motivation variables measured in the present study.

Another potential explanation could relate to the unclear and unmet needs of the older adults who were participating in this pilot study. An initial enthusiastic feeling about being part of the pilot study and being able to walk more independently could have been followed by an observation that the Smart Rollator did not entirely fulfill their expectations. As revealed in the interviews, disappointment or even frustration arose if the user was not able to fully benefit from the Smart Rollator as expected. However, this issue was not addressed during the interviews and, thus, should be studied further. Further, because MQ did not differentiate between user groups, we do not know to what extent this finding is specifically related to the intelligent features of the Smart Rollator. This single finding might even be incidental or related to the small sample size because the total motivation score revealed no difference.

However, the intelligent features granted additional value for some rollator users according to their assessments and descriptions, which became visible through the three Smart Rollator participant user types. Altogether, these findings support the future development of the Smart Rollator so that individual life situations, competencies, and needs can be more fully addressed. Based on the variation of the reported experiences of the participants, the intelligent features could be tailored to better fit the needs and practical demands of the users. The Smart Rollator could have a wider range of features from which the most expedient ones could be selected for the user. This customization could potentially reduce the frustration and experiences of disappointment, thus increasing usability. Further, tailoring of the features based on the individual might even benefit common therapeutic goals in the therapist relationship.
The present pilot study showed that intelligent features in the rollator granted additional value for some users. The whole care setting must account for the special requirements of older adults, the requirements of the significant others of the older adults, and care personnel. Mere technological solutions are insufficient without understanding the needs of the users.

However, it is notable that the individual variation was large, and some users reported clear advantages provided by the Smart Rollator's features. It also appeared that the Smart Rollator caused strong emotional reactions, such as affection or even frustration if the user was not able to benefit from the Smart Rollator as expected. In a dissemination event after the study in which one of the users presented his experiences, it turned out that his wife had been more interested in the movement data, encouraging her husband to move more. Thus, the use of rollators with intelligent features might increase the extrinsic motivation in older adults through the influence of relatives and caregivers.

Studies within Internet learning have shown that older users may believe they have the low technical competence and, thus, may lack selfconfidence (e.g., Chiu \& Liu, 2017). A lack of sufficient technical skills, confidence, and courage became visible in the experiences of disappointed users in the current study. The negative emotions experienced because of the technical problems prohibited their usage and learning of the mobile application. Thus, the criteria for technology as usable, easily accessible, and offering positive emotions triggering learning (Lee \& Coughlin, 2015) were not fully met by these users. From the perspective of pedagogical guidance, the existence of technical and social support is of great importance in helping older users solve the problems they encounter.

Further, research has indicated that interfaces that do not meet the practical needs of older users may increase the difficulties faced in terms of technology learning (Czaja et al., 2006; Huber \& Watson, 2014). Thus, meaningful learning activities that meet the everyday needs of older adults need to be considered when developing rollators with intelligent features in the future (see also Martins et al., 2015).

In concordance with prior research (e.g., Hill, Betts, \& Gardner, 2015), the results of the current pilot study highlight the individual variation in the needs, use purposes, and competences of older adults in adopting and taking advantage of intelligent technology in their everyday lives. This differentiation sets future challenges for research on older adults' experiences of intelligent technology 
and for the development of such technology.

Personalized medicine is rapidly having an impact on how patients are diagnosed and treated and how health care delivery is channeling its resources to maximize patient benefits (Pritchard et al., 2017). More individual solutions need to be found to promote healthy behavior, such as taking care of everyday physical activity like walking and, above all, the empowerment of the patient. New technologies such as the Smart Rollator may be significant contributors in that respect. It may also help to identify the most suitable patients for technical solutions like the Smart Rollator.

The limitations of the current study include the use of the unvalidated $\mathrm{MQ}$ and the small sample size. In future studies, a larger sample, a combination of several physical and behavioral measures, and a longer follow-up period could prove to be efficient. A shortcoming of the present study is that the majority of participants were women, with only one male participant in both user groups. Our data do not provide any explanation for this gender distribution skew. All individuals aged 60 and over with a need for continuous use of a rollator were offered the opportunity to participate in the study, and coincidentally, only one male in each user group was included. Plausible here is the huge gap in favor of women when it comes to life expectancy at birth in Finland (Ek, 2015), which could partly explain the difference between males and females of this age (mean ages of 80 and 81 in the user groups, respectively).

A strength of the current pilot study is the subjective user information measured using both quantitative and qualitative data collection methods, with the credibility of the results lying in the different types of users described regarding subjec- tive attitudes. These findings can help improve usability by tailoring the intelligent features of rollators with an emphasis on the demands and needs of older adults.

\section{Conclusions}

The intelligent features neither affected the motivation of users to use the rollator nor their experiences in doing so. The participants described a range of experiences with intelligent features, and the user types identified differed from each other, especially in terms of intelligent technology experiences. Those with previous experience with rollators were more eager to utilize the feedback from the Smart Rollator and reported that the intelligent features increased their motivation to move more. In contrast, the first-time rollator users did not find the intelligent features that meaningful; instead, they were adjusting to a new life situation accompanied by rollator aid. Overall, the experiences highlighted the central meaning of the prior experience and skills of using intelligent technology to benefit from the intelligent features of the Smart Rollator. Thus, the results of the current study provide insights into the further development of rollators with intelligent features in a way that acknowledges prior familiarity and experience - or a lack of experience - both with a rollator and with the intelligent features of the rollator. Mere technological solutions are insufficient without understanding the needs of the users and possible use purposes. Based on the findings, we argue that more weight should be put on guidance and support for older adults using Smart Rollators in their everyday lives. To foster older adults' coping in a new life situation and a need to learn new technological skills, support efforts should consider the various expectations, needs, prior experiences, and skills of older adults.

\section{Acknowledgements}

The research project received the approval (HUS/491/2018) of the Ethical Committee of Helsinki University Hospital (HUS). Each participant was given an information form, and the research assistant read it to them and clarified the course of the study, after which they provided their consent to participate.

This project was financed by the Academy of Finland (313898). We thank the occupational therapists Johanna Kuisma and Salla Tahkolahti at Laakso Hospital, Helsinki, Finland, for helping with the recruitment of participants. We warmly thank psychologist Esko Silén for his help in structuring and conducting qualitative interviews.

An oral presentation of the results of the study has been provided: Paloniemi S. "Does Smart Rollator support the daily lives of elderly adults?" At research days for adult education. Rovaniemi, Finland 13-14 February 2020.

\section{References}

Andersen, Z. J., De Nazelle, A., Mendez, M. A., Garcia-Aymerich, J., Hertel, O., Tjønneland, A., ... \& Nieuwenhuijsen, M. J. (2015). A study of the combined effects of physical activity and air pollution on mortality in elderly urban residents: the Danish Diet, Cancer, and Health Cohort. Environmental Health Perspectives, 123(6), 557-563.

Bertrand, K., Raymond, M. H., Miller, W. C., Ginis, K. A. M., \& Demers, L. (2017). Walking aids for enabling activity and participation: a systematic review. American Journal of Physical Medicine \& Rehabilitation, 96(12), 894-903.

Bouaziz, W., Lang, P. O., Schmitt, E., Kaltenbach, G., Geny, B., \& Vogel, T. (2016). Health benefits of multicomponent training programmes in seniors: a systematic review. International Journal of Clinical Practice, 70(7), 520-536. 
Brandt, ̊̊., Iwarsson, S., \& Ståhl, A. (2003). Satisfaction with rollators among community-living users: a follow-up study. Disability and Rehabilitation, 25(7), 343-353.

Braun, V., \& Clarke, V. (2006). Using thematic analysis in psychology. Qualitative Research in Psychology, 3 (1), 77-101.

Briggs, A. M., Cross, M. J., Hoy, D. G., Sanchez-Riera, L., Blyth, F. M., Woolf, A. D., \& March, L. (2016). Musculoskeletal health conditions represent a global threat to healthy aging: a report for the 2015 World Health Organization world report on ageing and health. The Gerontologist, 56(suppl_2), S243-S255.

Chang, V. C., \& Do, M. T. (2015). Risk factors for falls among seniors: implications of gender. American Journal of Epidemiology, 181(7), 521-531.

Changizi, M., \& Kaveh, M. H. (2017). Effectiveness of the mHealth technology in improvement of healthy behaviors in an elderly population-A Systematic review. Mhealth, 3.

Chiu, C. J., \& Liu, C. W. (2017). Understanding older adult's technology adoption and withdrawal for elderly care and education: mixed method analysis from national survey. Journal of medical internet research, 19(11), e374.

Cocosila, M., Archer, N., \& Yuan, Y. (2009). Early investigation of new information technology acceptance: A perceived risk-motivation model. Communications of the Association for Information Systems, 25(1), 30.

Cruz, D. T. D., Vieira, M. D. T., Bastos, R. R., \& Leite, I. C. G. (2017). Factors associated with frailty in a community-dwelling population of older adults. Revista de Saude Publica, 51, 106.

Czaja, S. J., Charness, N., Fisk, A. D., Hertzog, C., Nair, S. N., Rogers, W. A., \& Sharit, J. (2006). Factors predicting the use of technology: Findings from the center for research and education on aging and technology enhancement (CREATE). Psychology and aging, 21(2), 333.

De Angeli, A., Cozza, M., Jovanovic, M., Tonolli, L., Mushiba, M., McNeill, A., ... \& Müller, C. (2016). Understanding Motivations in Designing for Older Adults. In 12th COOP-International Conference on the Design of Cooperative Systems.

Denkinger, M. D., Lukas, A., Nikolaus, T., \& Hauer, K. (2015). Factors associated with fear of falling and associated activity restriction in community-dwelling older adults: a systematic review. The American Journal of Geriatric Psychiatry, 23(1), 72-86.

Einbinder E, Horrom TA. Smart Walker: A tool for promoting mobility in elderly adults. The Journal of Rehabilitation Research and Development, 2010;47(9):xiii-xvi. DOI:10.1682/ JRRD.2010.08.0154

Ek, S. (2015). Gender differences in health information behaviour: a Finnish population-based survey. Health promotion international, 30(3), 736-745.

Farholm, A., \& Sørensen, M. (2016). Motivation for physical activity and exercise in severe mental illness: A systematic review of intervention studies. International Journal of Mental Health Nursing, 25(3), 194-205.

Glynn, S. M., Taasoobshirazi, G., \& Brickman, P. (2009). Science motivation questionnaire: Construct validation with nonscience majors. Journal of Research in Science Teaching: The Official Journal of the National Association for Research in Science Teaching, 46(2), 127-146.

Guay, F., Vallerand, R. J., \& Blanchard, C. (2000). On the assessment of situational intrinsic and extrinsic motivation: The Situational Motivation Scale (SIMS). Motivation \& Emotion, 24(3), 175-213.

Halaweh, H., Willen, C., Grimby-Ekman, A., \& Svantesson, U. (2015). Physical activity and health-related quality of life among community dwelling elderly. Journal of Clinical Medicine Research, 7(11), 845.

Hellström, T., Lindahl, O., Bäcklund, T., Karlsson, M., Hohnloser, P., Bråndal, A., Hu, X., \& Wester, P. (2016). An intelligent rollator for mobility impaired persons, especially stroke patients. Journal of Medical Engineering \& Technology, 40 (5), 270-279. doi: 10.3109/03091902.2016.1167973

Henry, A., \& Thorsen, C. (2018). Teacher-student relationships and L2 motivation. The Modern Language Journal, 102(1), 218-241.

Hill, R., Betts, L.R., \&Gardner, S.E. (2015). Older adults' experiences and perceptions of digital technology: (Dis)empowerment, wellbeing, and inclusion. Computers in Human Behavior, 48, 415-423.

Huber, L., \& Watson, C. (2014). Technology: Education and training needs of older adults. Educational Gerontology, 40(1), 16-25.

Hupin, D., Roche, F., Gremeaux, V., Chatard, J. C., Oriol, M., Gaspoz, J. M., ... \& Edouard, P. (2015). Even a low-dose of moderate-to-vigorous physical activity reduces mortality by $22 \%$ in adults aged $\geq 60$ years: a systematic review and meta-analysis. British Journal of Sports Medicine, 49(19), 1262-1267.

Lee, C., \& Coughlin, J. F. (2015). PERSPECTIVE: Older adults' adoption of technology: an integrated approach to identifying determinants and barriers. Journal of Product Innovation Management, 32(5), 747-759.

Majumder, S., Aghayi, E., Noferesti, M., MemarzadehTehran, H., Mondal, T., Pang, Z., \& Deen, M. J. (2017). Smart homes for elderly healthcare-Recent advances and research challenges. Sensors, 17(11), 2496.

Mansouri, N., \& Goher, K. (2016). Walking aids for older adults: review of end-user needs. Asian Social Science; Vol. 12, No. 12; 2016 ISSN 1911-2017 EISSN 1911-2025.

Martins, M., Santos, C., Frizera, A., \& Ceres, R. (2015). A review of the functionalities of smart walkers. Medical Engineering \& Physics, 37(10), 917-928.

McCambridge, J., Witton, J., \& Elbourne, D. R. (2014). Systematic review of the Hawthorne effect: new concepts are needed to study research participation effects. Journal of Clinical Epidemiology, 67(3), 267-277.

Morton, K., Beauchamp, M., Prothero, A., Joyce, L., Saunders, L., Spencer-Bowdage, S., ... \& Pedlar, C. (2015). The effectiveness of motivational interviewing for health behaviour change in primary care settings: a systematic review. Health Psychology Review, 9(2), 205-223.

Mostaghel, R. (2016). Innovation and technology for the elderly: Systematic literature review. Journal of Business Research, 69(11), 4896-4900. 
Mundt, M., Batista, J. P., Markert, B., Bollheimer, C., \& Laurentius, T. (2019). Walking with rollator: a systematic review of gait parameters in older persons. European Review of Aging and Physical Activity, 16(1), 15.

O'Neil, H. F., \& Drillings, M. (Eds.). (2012). Motivation: Theory and research. Routledge.

Parker, M. G., \& Thorslund, M. (2007). Health trends in the elderly population: getting better and getting worse. The Gerontologist, 47(2), 150-158.

Physical Activity Guidelines Advisory Committee. (2008). Physical activity guidelines advisory committee report, 2008. Washington, DC: US Department of Health and Human Services, 2008, A1-H14.

Postolache, O., Pereira, J. M. D., Viegas, V., Pedro, L., Girão, P. S., Oliveira, R., \& Postolache, G. (2015). Smart walker solutions for physical rehabilitation. IEEE Instrumentation \& Measurement Magazine, 18(5), 21-30.

Pritchard, D. E., Moeckel, F., Villa, M. S., Housman, L. T., McCarty, C. A., \& McLeod, H. L. (2017). Strategies for integrating personalized medicine into healthcare practice. Personalized medicine, 14(2), 141-152.

Reid, S. E., Abdulrazak, B., \& Alas, M. (2017). The Underlying Motivations for Mobile Device Use by Seniors. Journal of Health Science, 5, 152-157.

Reiss, S. (2004). Multifaceted nature of intrinsic moti- vation: The theory of 16 basic desires. Review of General Psychology, 8(3), 179-193.

Riessman, C. K. (2008). Narrative Methods for the Human Sciences. Los Angeles: SAGE.

Roman de Mettelinge, T., \& Cambier, D. (2015). Understanding the relationship between walking aids and falls in older adults: a prospective cohort study. Journal of Geriatric Physical Therapy, 38(3), 127-132.

Ryan, R. M. (Ed.). (2012). The Oxford Handbook of Human Motivation. OUP USA.

Sawa, R., Asai, T., Doi, T., Misu, S., Murata, S., \& Ono, R. (2020). The association between physical activity, including physical activity intensity, and fear of falling differs by fear severity in older adults living in the community. The Journals of Gerontology: Series B, 75(5), 953-960.

Vroman, K. G., Arthanat, S., \& Lysack, C. (2015). "Who over 65 is online?" Older adults' dispositions toward information communication technology. Computers in Human Behavior, 43, 156-166. https://doi.org/10.1016/j.chb.2014.10.018

World Health Organization. (2015). World report on ageing and health. World Health Organization.

Yusif, S., Soar, J., \& Hafeez-Baig, A. (2016). Older people, assistive technologies, and the barriers to adoption: A systematic review. International Journal of Medical Informatics, 94, 112-116. 